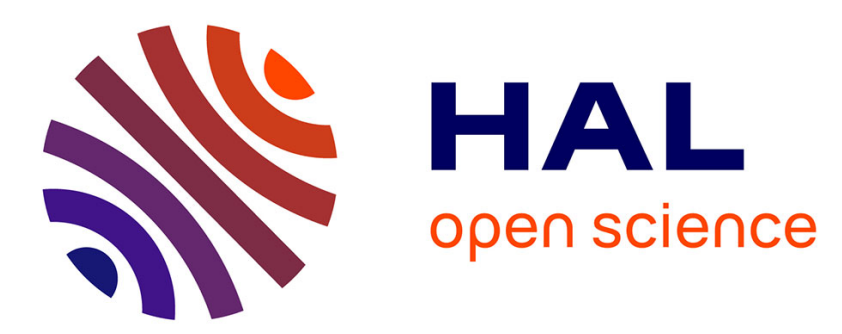

\title{
Joint Connectivity-Coverage Temperature-Aware Algorithms for Wireless Sensor Networks
}

Abdelmalik Bachir, Walid Bechkit, Yacine Challal, Abdelmadjid Bouabdallah

\section{To cite this version:}

Abdelmalik Bachir, Walid Bechkit, Yacine Challal, Abdelmadjid Bouabdallah. Joint ConnectivityCoverage Temperature-Aware Algorithms for Wireless Sensor Networks. IEEE Transactions on Parallel and Distributed Systems, 2015, 26 (07), pp.1923-1936. 10.1109/TPDS.2014.2331063 . hal$01006039 \mathrm{v} 2$

\section{HAL Id: hal-01006039 \\ https://hal.science/hal-01006039v2}

Submitted on 24 Dec 2015

HAL is a multi-disciplinary open access archive for the deposit and dissemination of scientific research documents, whether they are published or not. The documents may come from teaching and research institutions in France or abroad, or from public or private research centers.
L'archive ouverte pluridisciplinaire HAL, est destinée au dépôt et à la diffusion de documents scientifiques de niveau recherche, publiés ou non, émanant des établissements d'enseignement et de recherche français ou étrangers, des laboratoires publics ou privés. 


\title{
Joint Connectivity-Coverage Temperature-Aware Algorithms for Wireless Sensor Networks
}

\author{
Abdelmalik Bachir, Walid Bechkit, Yacine Challal Member, IEEE, Abdelmadjid Bouabdallah Member, IEEE
}

\begin{abstract}
Temperature variations have a significant effect on low power wireless sensor networks as wireless communication links drastically deteriorate when temperature increases. A reliable deployment should take temperature into account to avoid network connectivity problems resulting from poor wireless links when temperature increases. A good deployment needs also to adapt its operation and save resources when temperature decreases and wireless links improve. Taking into account the probabilistic nature of the wireless communication channel, we develop a mathematical model that provides the most energy efficient deployment in function of temperature without compromising the correct operation of the network by preserving both connectivity and coverage. We use our model to design three temperature-aware algorithms that seek to save energy (i) by putting some nodes in hibernate mode as in the SO (Stop-Operate) algorithm, or (ii) by using transmission power control as in PC (Power-Control), or (iii) by doing both techniques as in SOPC (Stop-Operate Power-Control). All proposed algorithms are fully distributed and solely rely on temperature readings without any information exchange between neighbors, which makes them low overhead and robust. Our results identify the optimal operation of each algorithm and show that a significant amount of energy can be saved by taking temperature into account.
\end{abstract}

Index Terms-Wireless Sensor Networks, Temperature Impact, Connectivity, Energy Saving.

\section{INTRODUCTION}

A wireless sensor network is composed of a number of battery operated nodes equipped with sensing, processing, storage, and wireless radio communication capabilities. These nodes are typically deployed in particular areas to monitor specific phenomena and report information to a central location or act accordingly. The deployments vary from application to another but generally nodes are unattended and are required to operate over long periods of time without external intervention or assistance. Therefore, nodes need to consume the least amount of energy while ensuring that the sensor network continues to operate correctly. In its basic form, the correct operation of the network can be achieved if both coverage and connectivity are preserved. The coverage ensures that the entire deployment area is monitored so that no event will be missed, and the connectivity means that the network is not fragmented so that data transmitted can reach any node in the network.

The correct operation of the network should be preserved constantly independently of changing conditions. Therefore, a good deployment should take into account the worst case conditions in which the network is also required to continue operating correctly. However, the worst case conditions may only happen in a limited number of occasions from time to time. In the rest of the time, the conditions are more favorable and the

Abdelmalik Bachir is with the CS Department, University of Biskra, Algeria, and the EEE Department, Imperial College London, UK. E-mail: a.bachir@imperial.ac.uk. Walid Bechkit is with Université de Lyon, INSALyon, CITI-INRIA, Villeurbanne, France. E-mail: walid.bechkit@insa-lyon.fr. Yacine Challal, Abdelmadjid Bouabdallah are with Université de Technologie de Compiègne, HEUDIASYC UMR CNRS 7253, Compiègne, France. Emails: \{ychallal, bouabdal\}@hds.utc.fr. network needs to adapt its operating mode to save more energy and resources. The detection of these situations needs to use small resources and be effective so that the advantages of adaptation are not surpassed by its overhead.

Temperature is one of those conditions that affect sensor networks significantly, particularly the quality of the wireless links between sensor nodes. In fact, sensor nodes are equipped with low-cost and low-power transceivers whose performance depends on the temperature in which they operate. Independent research results (see Section 2) and data taken from the data sheets of popular radio transceivers such as CC2400 [1], CC2530 [2] show that temperature affects both the quality of transmission and reception. As shown in Fig. 1, when temperature increases, both transmission power and reception sensitivity decrease ${ }^{1}$ thereby leading to the degradation of wireless communication links between transmitters and receivers. For example, the wireless links of a network designed to operate under typical conditions, i.e. a temperature of $25^{\circ} \mathrm{C}$, will experience degradations when temperature increases above $25^{\circ} \mathrm{C}$, which affects the correct operation of the entire network. Conversely, when temperature decreases below $25^{\circ} \mathrm{C}$, the quality of wireless links improves and the network becomes over-dimensioned. In this case, nodes may be able to optimize their operations (e.g. by using smaller transmission power) to save energy while still preserving the correct operation of the network.

In this paper, we propose to study the global effect of temperature on the correct operation of the network

1. The sensitivity decreases when the required received power to decode the signal increases. 


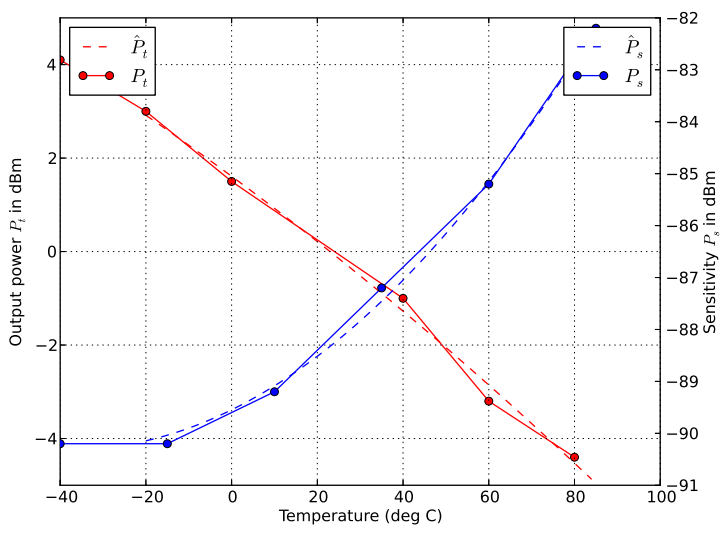

Fig. 1. Effect of temperature variations on the transmit power $P_{t}$ (nominal target of $0 \mathrm{dBm}$ ) and sensitivity $P_{s}$ of the CC2400. $P_{t}$ and $P_{s}$ are taken from the data sheet of CC2400 [1], and $\hat{P}_{t}$ and $\hat{P}_{s}$ are generated by polynomial interpolation with a polynomial degree equal to 2 .

in stable radio environments. We propose to adapt the network to the changing temperature so that minimum energy is consumed, without affecting its correct operation by preserving both connectivity and coverage. The key aspect of temperature is that link quality estimation can be achieved only by measuring temperature, which avoids the costly need for exchanging messages to assess link quality. Even though there may be some occasions where radio links change due to transient phenomena, we argue that in general the topology remains stable particularly in some environments.

We provide a set of low overhead fully distributed algorithms designed to operate correctly under extreme conditions where temperature is at maximum level and adapt the operation mode when temperature decreases or increases so that only a small amount of energy is consumed. We explore three different algorithms: (i) one that operates by putting nodes in hibernate mode, (ii) another one that makes nodes reduce their transmission power, and (iii) a combination of those two techniques. Our algorithms can be viewed as topology control algorithms as they operate by changing the topology, however, they fundamentally differ from traditional algorithms by their minimum overhead cost which consists in measuring temperature from time to time to make adaptation decisions. A comparison with other topology control algorithms is summarized in the related work section (see Section 7). The main contributions of the paper are the following:

- a model that describes the variation of connectivity of the network in function of temperature

- a closed formula that provides the minimum density of nodes required to achieve coverage and connectivity in function of temperature

- a low-overhead energy saving joint connectivity- coverage preserving algorithms in stable radio environments governed by deterministic changes such as those caused by temperature variations.

The rest of the paper is organized as follows. In Section 2 , we present a literature review of temperature-aware protocols, and show that little research has been done on evaluating the global effect of temperature on wireless sensor network. In Section 3, we present our analytical model for wireless links, connectivity and correct operation of the network. In Section 4, we show the effect of temperature on global network metrics, namely connectivity and coverage. In Section 5, we propose three energy saving algorithms that react to temperature changes, and evaluate their performance in Section 6 . In Section 7, we discuss the analogy between our algorithms and state-of-the-art network topology control techniques. We conclude in Section 8.

\section{Temperature-Aware Protocols Re- LATED WORK}

The effect of weather conditions such as rain, snowfall, wind, humidity and temperature on the performance of wireless communication has attracted the interest of a constant research work.

Early work on weather effect on wireless communication has been carried out in [3] which showed that the effect of temperature is among the most significant compared to other weather conditions. The authors of [3] showed that high temperatures affect the transmitter by causing distortion in the output waveform during final stage amplification thereby resulting in a poor signal being transmitted.

The effect of temperature on wireless communications has also been studied [4] and [5] in the context of sensor networks. The authors of [4] and [5] showed through experiments that temperature does not have effect on the antenna but rather affects the PA (Power Amplifier) at the transmitter and the LNA (Low Noise Amplifier) at the receiver, which results in poor links. They also showed that the RSSI (Received Signal Strength Indicator) noise floor decreases with temperature and highlighted the important effect of temperature on MAC protocols as these use the RSSI noise floor to assess whether the communication channel is free or busy. They also showed by experimenting with two different platforms, Tmote Sky using CC2420 (running at 2.4GHz) and the MSB 430 using CC1020 (running at $868 \mathrm{MHz}$ ), that temperature dependency exists on two different frequencies. As a way to cope with temperature changes in an industrial deployment, they proposed a protocol that adapts transmission power in function of temperature.

Another work on the effect of temperature on wireless links can be found in [6] where the authors examined the change of the link quality according to temperature through empirical experimentation. They proposed a power control scheme combining both temperature- 
aware link quality compensation and a closed-loop feedback process to adapt to link quality changes.

In [7], the effect of temperature on wireless links has been studied experimentally in the context of sensor networks. The authors of [7] quoted a decrease by $3.5 \mathrm{~dB}$ of the signal strength when temperature increases from $20^{\circ} \mathrm{C}$ to $50^{\circ} \mathrm{C}$. To cope with this effect, they proposed a transmission power control algorithm that aims at maintaining wireless links while saving energy consumption by using the minimum transmission power that preserves an LQI higher than a certain threshold.

Beyond the effect of temperature on individual links, the effect of temperature on other global metrics such as connectivity has been studied in [8], also in the context of sensor networks. The authors of [8] also showed that temperature affects both transmitter and receiver and quoted a decrease by $5 \mathrm{~dB}$ of the measured power at the transmitter and a decrease in the receiver sensitivity by $3 \mathrm{~dB}$ over the $40^{\circ} \mathrm{C}$ range. They also studied, by simulation, the effect of temperature on network connectivity, data collection and localization and recommended that existing protocols should be improved to take into account the effect of temperature.

Most of the work, as shown above, has focused on experimentally quantifying the effect of weather conditions on wireless sensor networks at the link level and considered typical metrics such as signal strength and link quality indicator (see [9] for link quality assessment techniques). Significantly less work has been done on studying the effect of those conditions on more general aspects such as global connectivity and coverage of the network, and even the existing work we are aware of (e.g. [8]) only studied the effect on connectivity by simulation. In this paper, we tackle these issues and study the effect of temperature, in particular, on both connectivity and coverage by providing mathematical fundamentals governing those effects. We also propose three different algorithms to allow wireless sensor networks to adapt to temperature variations to ensure the network continues to operate correctly while consuming lesser energy.

\section{SYSTEM MODEL}

\subsection{Wireless Links}

The quality of a wireless communication link between two nodes, a transmitter and a receiver, is usually expressed by the experienced PER (Packet Error Rate). The PER mainly depends on the signal strength measured at the receiver. The minimum signal strength measured at the receiver required to achieve a PER of less than $1 \%$ is called sensitivity. We assume that a link exists if the received power $P_{r}$ is equal to or larger than the sensitivity $P_{s}$. The signal strength measured at the receiver depends on the transmission power $P_{t}$ radiated by the transmitter and its attenuation along the communication channel. The attenuation $\mathcal{A}(d)$ over a distance $d$ is the ratio between $P_{t}$ and $P_{r}(d)$, where $P_{r}(d)$ is the power of the signal received at distance $d$ from the transmitter. When
$P_{t}$ and $P_{r}(d)$ are expressed in $\mathrm{dBm}$, the attenuation in $\mathrm{dB}$ is:

$$
\mathcal{A}(d)=P_{t}-P_{r}(d)
$$

A commonly used model for the attenuation is the lognormal path loss model [10], where the attenuation has three components: $\mathcal{A}_{0}, \mathcal{A}_{1}(d)$ and $\mathcal{A}_{2}$ such that:

$$
\mathcal{A}(d)=\mathcal{A}_{0}+\mathcal{A}_{1}(d)+\mathcal{A}_{2}
$$

where $\mathcal{A}_{0}$ is the path loss at reference distance $d_{0}$, which can be obtained by experimentation, or according to the free space propagation model, $\mathcal{A}_{1}(d)$ is the log-distance path loss in function of distance $d$ and reference distance $d_{0}$, and $\mathcal{A}_{2}$ is a random variable reflecting the attenuation caused by fading. All $\mathcal{A}_{0}, \mathcal{A}_{1}$, and $\mathcal{A}_{2}$ are expressed in $\mathrm{dB}$. By taking $\mathcal{A}_{0}$ according to the free space propagation model, we obtain:

$$
\begin{cases}\mathcal{A}_{0} & =20 \log d_{0}+20 \log f-27.55 \\ \mathcal{A}_{1}(d) & =\alpha 10 \log \left(d / d_{0}\right) \\ \mathcal{A}_{2} & \sim \mathcal{N}(0, \sigma)\end{cases}
$$

where $f$ is the frequency (in $\mathrm{MHz}$ ), $\alpha$ is the path loss exponent, and $\mathcal{N}(0, \sigma)$ is a normal random variable (in $\mathrm{dB})$. We set the reference distance $d_{0}$ to be equal to $1 \mathrm{~m}$.

\subsection{Communication Range}

We define the communication range as the distance at which less than $1 \%$ of packets are lost (i.e. corresponding to less than $1 \%$ of PER). The communication range is a random variable $\mathcal{R}$ with the cumulative distribution function $F_{\mathcal{R}}(d)$ defined as:

$$
\begin{aligned}
F_{\mathcal{R}}(d) & =\operatorname{Pr}[\mathcal{R} \geq d]=\operatorname{Pr}\left[P_{r}(d) \geq P_{s}\right] \\
& =\operatorname{Pr}\left[P_{t}-P_{r}(d) \leq P_{t}-P_{s}\right] \\
& =\operatorname{Pr}\left[\mathcal{A}(d) \leq P_{t}-P_{s}\right]
\end{aligned}
$$

By replacing $\mathcal{A}(d)$ by its value from (2), we obtain:

$$
F_{\mathcal{R}}(d)=\operatorname{Pr}\left[\mathcal{A}_{2} \leq P_{t}-P_{s}-\mathcal{A}_{0}-\mathcal{A}_{1}(d)\right]
$$

As $\mathcal{A}_{2}$ is a normal random variable, we have:

$$
\begin{aligned}
\operatorname{Pr}\left[\mathcal{A}_{2} \leq P_{t}-P_{s}-\right. & \left.\mathcal{A}_{0}-\mathcal{A}_{1}(d)\right]= \\
& F_{\mathcal{A}_{2}}\left(P_{t}-P_{s}-\mathcal{A}_{0}-\mathcal{A}_{1}(d)\right)
\end{aligned}
$$

where $F_{\mathcal{A}_{2}}$ is the CDF of the random variable $\mathcal{A}_{2}$. As $\mathcal{A}_{2} \sim \mathcal{N}(0, \sigma)$, we have:

$$
\begin{aligned}
F_{\mathcal{A}_{2}}\left(P_{t}-P_{s}-\right. & \left.\mathcal{A}_{0}-\mathcal{A}_{1}(d)\right)= \\
& \frac{1}{2}+\frac{1}{2} \operatorname{erf}\left(\frac{P_{t}-P_{s}-\mathcal{A}_{0}-\mathcal{A}_{1}(d)}{\sigma \sqrt{2}}\right)
\end{aligned}
$$

where erf is the error function defined as:

$$
\operatorname{erf}(z)=\frac{2}{\sqrt{\pi}} \int_{0}^{z} e^{-t^{2}} d t
$$

By combining (5), (6) and (7), we get:

$$
F_{\mathcal{R}}(d)=\frac{1}{2}+\frac{1}{2} \operatorname{erf}\left(\frac{P_{t}-P_{s}-\mathcal{A}_{0}-\mathcal{A}_{1}(d)}{\sigma \sqrt{2}}\right)
$$


Note that (7) and (9) are only valid when $\sigma \neq 0$. When $\sigma=0, \mathcal{A}_{2}$ becomes equal to 0 . Therefore, the expression of $F_{\mathcal{R}}(d)$ should be calculated from (5). Thus, we have:

$$
\begin{aligned}
F_{\mathcal{R}}(d)_{\sigma=0} & =\operatorname{Pr}\left[0 \leq P_{t}-P_{s}-\mathcal{A}_{0}-\mathcal{A}_{1}(d)\right] \\
& =\operatorname{Pr}\left[\mathcal{A}_{1}(d) \leq P_{t}-P_{s}-\mathcal{A}_{0}\right]
\end{aligned}
$$

By replacing $\mathcal{A}_{1}(d)$ with its value from (3) and taking $d_{0}=1$, (11) becomes:

$$
\begin{aligned}
F_{\mathcal{R}}(d)_{\sigma=0} & =\operatorname{Pr}\left[10 \alpha \log d \leq P_{t}-P_{s}-\mathcal{A}_{0}\right] \\
& =\operatorname{Pr}\left[d \leq 10^{\left(P_{t}-P_{s}-\mathcal{A}_{0}\right) /(10 \alpha)}\right] \\
& = \begin{cases}1 & \text { if } d \leq 10^{\left(P_{t}-P_{s}-\mathcal{A}_{0}\right) /(10 \alpha)}, \\
0 & \text { elsewhere. }\end{cases}
\end{aligned}
$$

By putting,

$$
R \triangleq 10^{\left(P_{t}-P_{s}-\mathcal{A}_{0}\right) /(10 \alpha)}
$$

and combining (9) with (14), we obtain:

$F_{\mathcal{R}}(d)= \begin{cases}\frac{1}{2}+\frac{1}{2} \operatorname{erf}\left(\frac{P_{t}-P_{s}-\mathcal{A}_{0}-\mathcal{A}_{1}(d)}{\sigma \sqrt{2}}\right) & \text { if } \sigma>0, \\ 1_{(-\infty, R]}(d) & \text { if } \sigma=0 .\end{cases}$

where $1_{(-\infty, R]}(d)$ is the indicator function, which is equal to 1 when $d \leq R$ and to 0 elsewhere. Note that $R$ represents the communication range in the absence of shadowing fading (i.e. when $\sigma=0$ ).

Note that the previous derivations also apply to the two ray ground model which may be more appropriate for outdoor environments. The results for the two ray ground model can be obtained by taking $\mathcal{A}_{0}=$ $10 \log \left(G_{t} G_{r} h_{t}^{2} h_{r}^{2}\right), \alpha=4$, and $\sigma=0$ in (3), where $G_{t}$ (resp. $G_{r}$ ) is the antenna gain at the transmitter (resp. receiver), and $h_{t}$ (resp. $h_{r}$ ) is the antenna height at the transmitter (resp. receiver).

\subsection{Connectivity and Coverage}

We consider the case where nodes are deployed according to a PPP (Poisson Point Process). Under these circumstances, the probability $q$ that the deployment area is fully covered can be approximated to the following [11]:

$$
q=1-e^{-\lambda \pi R_{v}^{2}}
$$

where $\lambda$ is the network density expressed by the number of nodes per unit area, and $R_{v}$ is the coverage range where all events occurring at a distance smaller than $R_{v}$ are covered (i.e. can be sensed) by the node.

The probability $p$ that the network is connected has been calculated in [12]. By putting,

$$
K \triangleq \pi e^{(\sqrt{2} \sigma / \alpha)^{2}}
$$

the probability $p$ can be written as follows:

$$
p=e^{-\lambda \mu e^{-\lambda K R^{2}}}
$$

where $\mu$ is the surface of the deployment area.

\subsection{Correct Operation of the network}

We assume that the network continues to operate correctly if the connectivity probability $p$ and the coverage probability $q$ are both larger or equal than preset values $p^{*}$ and $q^{*}$, respectively. Typically $p^{*}$ and $q^{*}$ can be taken equal to 0.99 . The minimum network density $\lambda_{v}$ required to achieve a given coverage probability $q$ can be calculated by rewriting (16). Therefore, we have:

$$
\lambda_{v}=\frac{-\ln (1-q)}{\pi R_{v}^{2}}
$$

Similarly, the minimum density $\lambda_{c}$ required to achieve a given connectivity probability $p$ can be calculated by rewriting (18) as follows:

$$
\begin{aligned}
\ln p & =-\lambda_{c} \mu e^{-\lambda_{c} K R^{2}} \\
\frac{K R^{2} \ln p}{\mu} & =-\lambda_{c} K R^{2} e^{-\lambda_{c} K R^{2}}
\end{aligned}
$$

By putting $Z \triangleq \frac{K R^{2} \ln p}{\mu}, z \triangleq-\lambda_{c} K R^{2},(21)$ becomes:

$$
Z=z e^{z}
$$

The solution to (22) is:

$$
z=W(Z)
$$

where $W($.$) is the Lambert \mathrm{W}$ function (see [13] for some properties). By replacing $Z$ and $z$ by their definition, we obtain the following:

$$
\lambda_{c}=\frac{-1}{K R^{2}} W\left(\frac{K R^{2} \ln p}{\mu}\right)
$$

By targeting $p^{*}$ and $q^{*}$ values for connectivity and coverage, the minimum required network density $\tilde{\lambda}$ is given by the following expression:

$$
\tilde{\lambda}=\max \left\{\frac{-1}{K R^{2}} W\left(\frac{K R^{2} \ln p^{*}}{\mu}\right), \frac{-\ln \left(1-q^{*}\right)}{\pi R_{v}^{2}}\right\}
$$

\section{Effect of Temperature}

\subsection{On Transmission Power}

We use the notation $P_{t, \tau}(I)$ to describe the transmission power in function of the temperature $\tau$ measured at the transmitter when an input current $I$ is used. Although the effect of temperature on the transmission power has been studied in the literature and documented in the data sheets, available data only show the variation of the transmission power in the typical case (i.e. where a nominal transmission power of $0 \mathrm{dBm}$ is targeted). We use the notation $I^{*}$ to refer to the typical input current, i.e. the one that is used to target typical transmission power of $0 \mathrm{dBm}$. Therefore, available results only show the variations of $P_{t, \tau}\left(I^{*}\right)$. Also, available results on transmission power control provide that variations of the transmission power in function of the input current in the typical case for temperature, i.e. $\tau$ around $25^{\circ} \mathrm{C}$, therefore values for $P_{t, \tau^{*}}(I)$ are also known, where $\tau^{*}$ is the typical temperature. 


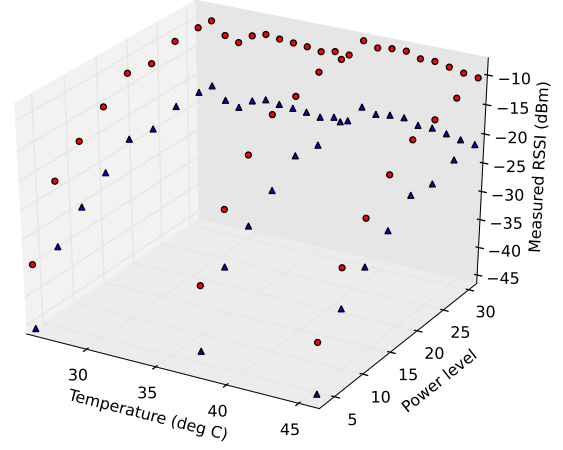

Fig. 2. The variation of the transmission power $P_{t, \tau}(I)$ CC2420. The triangles are generated numerically according to the assumption that we set in (26) and the circles are the result of experimentation.

To capture variation of $P_{t, \tau}(I)$ in the general case, we assume that the shape ${ }^{2}$ of the variation in function temperature would be the same for all input currents, i.e. the effects of temperature and input current on the output power are additively separable in $\mathrm{dBm}$, which can be written as:

$$
P_{t, \tau}(I)=P_{t, \tau}\left(I^{*}\right)+P_{t, \tau^{*}}(I)
$$

\subsection{Validation by Experiments}

To validate the assumption in (26), we run a set of experiments where we vary both temperature and the input current at the transmitter and we measure the signal strength at the receiver, i.e. for each temperature $\tau$ and input current $I$, we measure $P_{r, \tau}(I)$. The transmission and reception powers are related to each other according to the equation described in (2), i.e. $P_{t, \tau}(I)=P_{r, \tau}(I)+a$ where $a$ is the attenuation, we can consider as constant during the experiments as we do not change the distance between the transmitter and the receiver. Therefore, validating the assumption in (26) reduces to validating that:

$$
\begin{aligned}
P_{r, \tau}(I)+a & =P_{r, \tau}\left(I^{*}\right)+a+P_{r, \tau^{*}}(I)+a \\
P_{r, \tau}(I) & =P_{r, \tau}\left(I^{*}\right)+P_{r, \tau^{*}}(I)+a
\end{aligned}
$$

We collect a large set of data for temperatures ranging from 26 to 54 and input currents ranging from $8.5 \mathrm{~mA}$ (to output a power of $-25 \mathrm{dBm}$ ) to $17.4 \mathrm{~mA}$ (to output a power of $0 \mathrm{dBm}$ ) (see Fig. 2). The values of $\tau^{*}$ and $I^{*}$ are equal to $26^{\circ} \mathrm{C}$ and $0 \mathrm{dBm}$, respectively. To validate our

2. If we take CC2400 radio transceivers as example, this assumption means that given $P_{t, \tau^{*}}(I)$, the output power at $38^{\circ} \mathrm{C}$ (resp. $50^{\circ} \mathrm{C}$ ) can be obtained by shifting the given graph at $25^{\circ} \mathrm{C}$ by $-1 \mathrm{dBm}$ (resp. $-2 \mathrm{dBm}$ ) and so on.

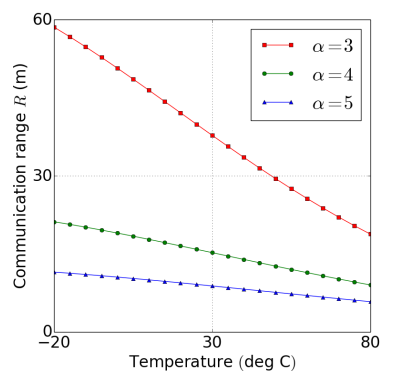

(a)

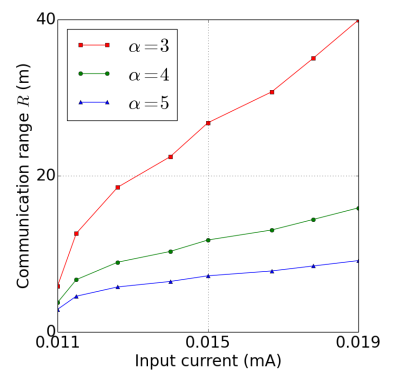

(b)
Fig. 3. (a) describes the effect of temperature on wireless links with various path loss exponents. The radio used is the CC2400 with a targeted transmission power of $0 \mathrm{dBm}$ corresponding to an input current of $19 \mathrm{~mA}$. (b) describes the effect of input current on wireless links with various path loss exponents. The radio used is the CC2400 with with a temperature of $25^{\circ} \mathrm{C}$.

linear model, we run a multidimensional regression on the collected data for $P_{r, \tau}(I)$. The general multidimensional regression expression is the following:

$$
P_{r, \tau}(I)=r_{1} P_{r, \tau}\left(I^{*}\right)+r_{2} P_{r, \tau^{*}}(I)+r_{3}
$$

The results of the multidimensional regression show that $r_{1}=1.05, r_{2}=1.06$, and $r_{3}=12.38$ with a high score of 0.98 ( 1 is the perfect score). These results validate our assumption in (26) as both $r_{1}$ and $r_{2}$ can be grossly approximated to 1 and $r_{3}$ represents the attenuation of the channel according to the channel between the transmitter and the receiver during our experiments.

\subsection{On Receiver Sensitivity}

As shown in the data sheets, the sensitivity of the receiver varies in function of temperature (see Figure 1). However, the current used during reception remains constant independently on the power strength received, thus we have the notation $P_{s, \tau}$ to express sensitivity in function of temperature.

\subsection{On Sensing}

There is a wide array of sensing devices and the effect of temperature on their performance may vary from a sensor to another. For the sake of simplicity, we assume in our study that the sensing range (also referred to as coverage range hereafter) is constant and does not depend on temperature variations, nor does it depend on input current. The study can be easily generalized when the function governing the variation of the coverage range $R_{v}$ in function of temperature $\tau$ is known. 


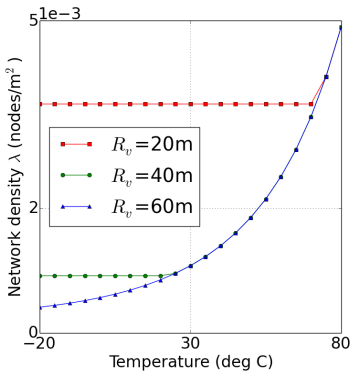

(a)

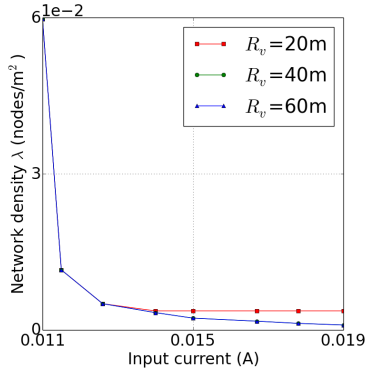

(b)
Fig. 4. (a) describes the minimum densities required to guarantee correct operation of the network. The communication range is calculated with $\alpha=3$. (b) describes the minimum densities required to guarantee correct operation of the network. The communication range is also calculated with $\alpha=3$.

\subsection{On Connectivity and Coverage}

The combination of transmission power and receiver sensitivity defines the communication range. Therefore, for the same temperature measured at the transmitter and the receiver, we use the notation $R_{\tau}(I)$. Fig. 3(a) and Fig. 3(b), plotted with values from $\hat{P}_{t}$ and $P_{s}$ (see Fig. 1), show the variations of the communication range $R_{\tau}(I)$ in function of temperature and input current, respectively. It shows that the reduction is more significant in environments with smaller path loss, the smaller the path loss the more significant the effect of temperature.

The minimum node density required to guarantee correct operation of the network depends on the transmission range, which in turn depends on temperature and input current. For an input current $I$ at temperature $\tau$, the minimum required density is $\tilde{\lambda}_{\tau}(I)$ which can be obtained directly from (25) by making $R$ depend on $\tau$ and $I$. Thus, we have:

$\tilde{\lambda}_{\tau}(I)=\max \left\{\frac{-1}{K R_{\tau}^{2}(I)} W\left(\frac{K R_{\tau}^{2}(I) \ln p^{*}}{\mu}\right), \frac{-\ln \left(1-q^{*}\right)}{\pi R_{v}^{2}}\right\}$

Fig. 4(a) and Fig. 4(b) show the evolution of the minimum network density that is required to maintain the correct operation of the network in function of temperature and input current variations, respectively.

\section{Temperature-Aware Energy Saving PROTOCOLS}

To guarantee the correct operation of the network in all circumstances, the number of deployed nodes need to be large enough so that the density of the initial deployment ensures that both connectivity and coverage are maintained. The worst case occurs when temperature is at maximum. If the initial deployment targets minimizing the number of nodes then all nodes will be transmitting with maximum power in the worst case. In these circumstances the initial deployment network density $\lambda_{0}$ is equal to the following:

$$
\lambda_{0}=\tilde{\lambda}_{\tau^{+}}\left(I^{+}\right)
$$

where $\tau^{+}$is the maximum temperature that is expected to be measured and $I^{+}$the maximum input current that can be used to transmit with maximum power. As temperature varies, the initial deployment envisaged for the worst case becomes over-dimensioned. We propose three algorithms to save energy.

- Stop-Operate (SO): a proportion of the initially deployed nodes is needed to guarantee the correct operation of the network and continue operating normally by using maximum transmission power. The rest of the nodes which are not required can stop operating and switch to hibernate mode to save energy

- Power-Control (PC): all nodes continue to operate normally but use lower transmission powers to save energy

- Combination of SO and PC (SOPC): a proportion of nodes is needed and those needed nodes may use lower transmission powers to save energy.

We assume that each node is able to know its temperature (temperature sensor readings, extraction from log files, computation, expectation, etc.) and that all nodes measure temperature at the beginning of each round. We also assume that there is no micro-climate, i.e. all nodes measure the same temperature.

Each one of the proposed algorithm is fully distributed; each node can run it without exchanging any additional information with its neighbors. Nodes only need to periodically measure temperature and take decisions locally. This requires a certain level of synchronization so that nodes measure temperature and run corresponding actions at nearly the same time. This relaxed synchronization can be maintained either by the periodic exchange of synchronization messages where (争Re period can be relatively long to save energy, or from the underlying MAC protocol if the protocol relies on synchronization such as the case in SCP-MAC [14] and IEEE 802.15.4 [15].

\subsection{Stop-Operate}

At temperature $\tau$ and input current $I$ only $\tilde{\lambda}_{\tau}(I)$ nodes per surface unit are needed for the correct operation of the network. We propose Algorithm 1 to make use of this result by allowing the proportion of nodes that are not necessary for the correct operation of the network to go to hibernate mode to save energy. We call $\rho_{\tau}(I)$ the operating ratio, which is the ratio of nodes that are operating normally at temperature $\tau$ and current $I$ compared to those initially deployed. We have:

$$
\rho_{\tau}(I)=\frac{\lambda_{\tau}(I)}{\lambda_{0}}
$$


For the correct operation of the network, we need that proportion to be equal to:

$$
\tilde{\rho}_{\tau}(I)=\frac{\tilde{\lambda}_{\tau}(I)}{\lambda_{0}}
$$

At the beginning of each round in Algorithm 1, each node generates a random number in $[0,1]$ that is used to make a decision. If the generated random number is greater than $\tilde{\rho}_{\tau}(I)$, the node can go to hibernate mode without affecting the correct operation of the network. However, if the generated number is smaller than or equal to $\tilde{\rho}_{\tau}(I)$, the node should be operating normally during the round, i.e. the node needs to resume operating normally if it was in hibernate mode, or continue operating normally if it was operating normally.

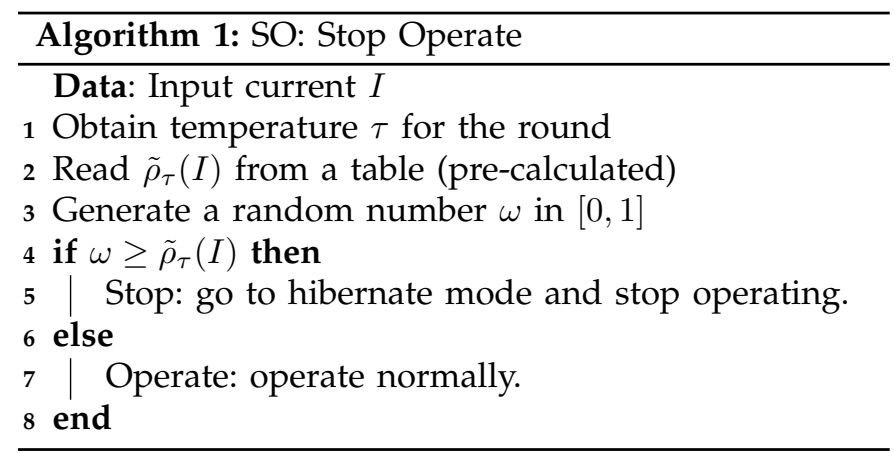

As shown in the Algorithm 1, the selection of those nodes which can stop operating can be done randomly. This is so because the distribution of nodes which continue to operate normally will maintain the same characteristics as the initial one, i.e. distributed according to PPP (see the thinning property [16]). For efficiency and practicality, the values of $\tilde{\rho}_{\tau}(I)$ for typical values of $\tau$ and $I$ can be stored in a table to avoid complex computation at the sensor node. The calculation of the values of $\tilde{\rho}_{\tau}(I)$ is straightforward, because the value of $\tilde{\lambda}_{\tau}(I)$ and $\lambda_{0}$ can be calculated from (30) and (31). These calculations can be done offline prior to deployment.

\subsection{Power Control}

In this case, we assume that nodes can use power control. As shown in previous sections, when temperature decreases, the wireless link quality improves and the using of smaller power transmission may be possible to save energy while maintaining the correct operation of the network. In the context of energy saving, using smaller transmission power may reduce the overall energy consumption of the network. Finding the optimal transmission power, i.e. finding the optimal input current, depends on whether the energy consumption model of the MAC protocol is known or not. When the model is unknown, we use the following heuristic: the smallest I that maintains the correct operation of the network will achieve the lowest energy consumption. The correct operation of the network will remain maintained if both connectivity and coverage are preserved. However, as all nodes deployed initially will continue to operate normally without experiencing effects on their coverage range, coverage will continue to be maintained independently of temperature variations. Therefore, it is sufficient to preserve connectivity (see (36)) to maintain the correct operation of the network. Therefore, we have:

$$
\begin{array}{rc}
\text { minimize } & I \\
\text { subject to : } & I \in\left\{I^{-}, \ldots, I^{+}\right\} \\
\text {and : } & p_{\tau}(I) \geq p^{*}
\end{array}
$$

Equation (36) can be rewritten as :

$$
\begin{aligned}
e^{-\lambda \mu e^{-\lambda K R_{\tau}(I)^{2}}} & \geq p^{*} \\
-\lambda \mu e^{-\lambda K R_{\tau}(I)^{2}} & \geq \ln p^{*} \\
e^{-\lambda K R_{\tau}(I)^{2}} & \leq-\ln p^{*} /(\lambda \mu) \\
-\lambda K R_{\tau}(I)^{2} & \leq \ln \left(-\ln p^{*} /(\lambda \mu)\right) \\
R_{\tau}(I)^{2} & \geq-\ln \left(-\ln p^{*} /(\lambda \mu) /(\lambda K)\right. \\
R_{\tau}(I) & \geq \sqrt{-\ln \left(-\ln p^{*} /(\lambda \mu)\right) /(\lambda K)} \\
\kappa^{P_{t, \tau}(I)-P_{s, \tau}} & \geq \sqrt{-\ln \left(-\ln p^{*} /(\lambda \mu)\right) /(\lambda K)} \\
P_{t, \tau}(I) & \geq P_{s, \tau}+Q
\end{aligned}
$$

where:

$$
Q \triangleq 10 \alpha \log \left(\sqrt{-\ln \left(-\ln p^{*} /\left(\lambda_{0} \mu\right)\right) /\left(\lambda_{0} K\right)}\right)
$$

The functions $P_{t, \tau}(I)$ and $P_{s, \tau}$ are usually provided as a discrete function giving the output power for each pre-defined input current and temperature. They may also be approximated by extrapolation. The constant $Q$ can be calculated prior to deployment or communicated to nodes from an external application when certain condition changes such as channel.

Since the number of pre-defined transmission levels is usually low in practice (eight in the case of CC2400), we propose the following Algorithm 2 to find the required transmission level in function of temperature.

\begin{tabular}{l}
\hline Algorithm 2: PC: Power Control \\
\hline 1 Obtain temperature $\tau$ for the round \\
$2 I \leftarrow I^{-}$(the smallest input current) \\
3 while not $\left(P_{t, \tau}(I)<P_{s, \tau}+Q\right)$ do \\
$4 \quad I \leftarrow$ the next input current level \\
5 end \\
6 Use input current $\tilde{I}=I$ for transmission \\
\hline
\end{tabular}

Note that the condition (44) which ensures that the network connectivity is preserved can be replaced by a more generic condition which includes both connectivity and coverage. In fact, it is sufficient that the density of the network is larger or equal to $\tilde{\lambda}$ calculated in (25). As we do not envisage adding more nodes than initially deployed, the density of the network cannot be larger 
than the initial $\lambda_{0}$, i.e. $\tilde{\lambda}_{\tau}(I) \leq \lambda_{0}$, i.e. $\tilde{\rho}_{\tau}(I) \leq 1$. In addition, $\tilde{\rho}_{\tau}(I)>0$; therefore, the condition (44) can be rewritten as:

$$
\tilde{\rho}_{\tau}(I) \in(0,1]
$$

\subsection{Combination of SO and PC}

In this section, we analyze the case where nodes combine the use of Stop-Operate with Power-Control. We assume that the energy consumption model is known. We use the notation $\mathcal{P}_{\tau}\left(I, \rho_{\tau}(I)\right)$ to refer to the mean power consumed by a node at temperature $\tau$ when nodes are using input current $I$ and when only a proportion of $\rho_{\tau}(I)$ of nodes is used. Therefore, when nodes are running Stop-Operate, a proportion of $\left(1-\rho_{\tau}(I)\right)$ will be in hibernate mode to save energy. The mean power consumption becomes equal to $\mathcal{Q}_{\tau}\left(I, \rho_{\tau}(I)\right)$ as follows:

$$
\mathcal{Q}_{\tau}\left(I, \rho_{\tau}(I)\right)=\rho_{\tau}(I) \mathcal{P}_{\tau}\left(I, \rho_{\tau}(I)\right)+\left(1-\rho_{\tau}(I)\right) \mathcal{P}_{\mathrm{h}}
$$

where $\mathcal{P}_{\mathrm{h}}$ is the mean power consumption at hibernate mode $^{3}$. As the energy model is known, the optimization problem becomes:

$$
\begin{array}{cc}
\operatorname{minimize} & \mathcal{Q}_{\tau}\left(I, \tilde{\rho}_{\tau}(I)\right) \\
\text { subject to : } & I \in\left\{I^{-}, \ldots, I^{+}\right\} \\
\text {and : } & \tilde{\rho}_{\tau}(I) \in(0,1]
\end{array}
$$

Therefore, we propose the following algorithm (Algorithm 3) which can be used when the energy model is known.

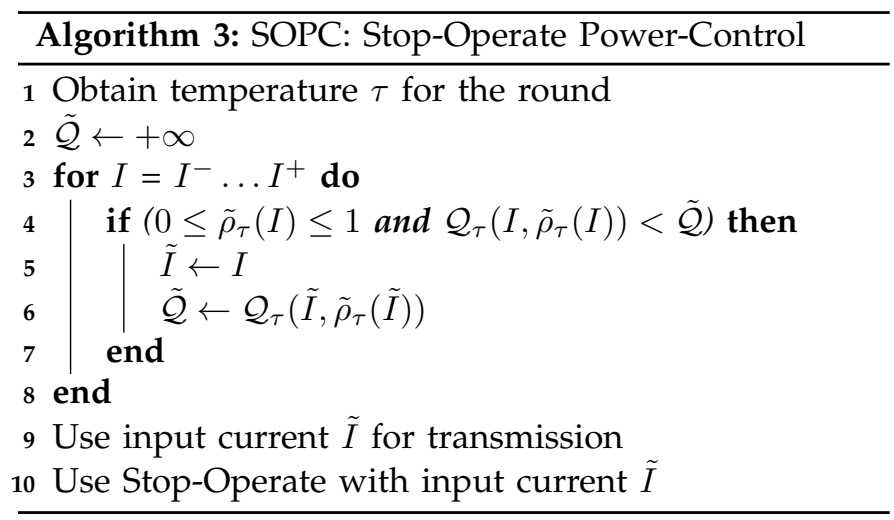

\section{Evaluation}

We use the analytical model developed in [14] for the evaluation of the energy consumption of the BMAC [17] and SCP-MAC [14] protocols. BMAC is one of the most deployed protocols in WSN as it is the default protocol in most of the sensor node prototypes (Micaz, Mica-2, etc.) that rely on the operating system TinyOs. BMAC

3. Note that $\mathcal{P}_{\mathrm{h}}$ is very small but not equal to zero, because the node

\begin{tabular}{|c|c|c|}
\hline \multicolumn{3}{|c|}{ Deployment } \\
\hline Deployment area & $\mu$ & $1000 \mathrm{~m} \times 1000 \mathrm{~m}$ \\
\hline Coverage range & $R_{v}$ & $200 \mathrm{~m}$ \\
\hline Coverage probability & $q$ & 0.99 \\
\hline Connectivity probability & $p$ & 0.99 \\
\hline Lognormal spread & $\sigma$ & 2 \\
\hline Path loss exponent & $\alpha$ & 3 \\
\hline \multicolumn{3}{|c|}{ MAC and Hardware } \\
\hline Voltage & $V$ & $3 \mathrm{v}$ \\
\hline Receive mode current consumption & $I_{r x}$ & $24 \mathrm{~mA}$ \\
\hline Sleep mode consumption & $P_{\text {sleep }}$ & $3 \mu \mathrm{W}$ \\
\hline Channel polling interval & $T_{p}$ & $200 \mathrm{~ms}$ \\
\hline Channel polling consumption & $P_{\text {poll }}$ & $12.3 \mathrm{~mW}$ \\
\hline Avg. Channel polling time & $t_{p l}$ & $2.5 \mathrm{~ms}$ \\
\hline Avg. Carrier sensing time & $t_{c s l}$ & $2 \mathrm{~ms}$ \\
\hline Byte transmission time & $t_{B}$ & $32 \mu \mathrm{s}$ \\
\hline Clock drift & $r_{c l k}$ & 30ppm \\
\hline \multicolumn{3}{|l|}{ Traffic } \\
\hline Data transmission interval & $T_{d a t a}$ & $300 s$ \\
\hline Data transmission frequency & $r_{d a t a}$ & $1.0 / T_{\text {data }} \mathrm{s}^{-1}$ \\
\hline Data packet length & $L_{\text {data }}$ & 50Bytes \\
\hline
\end{tabular}
keeps running essential components such as some timers to allow itself to resume operating normally.
TABLE 1

Default values of the parameters used in the analysis.

achieves high energy savings through the use of preamble sampling technique (see [18] for a survey on these protocols) where nodes spend most of their time in sleep mode, but only wake up from time to time to check for incoming traffic. To make sure that a node does not miss an incoming data packet, each node prepends its data packet transmission with a preamble that is long enough so that any potential receiver will wake up during the transmission of the preamble. When a node wakes up and detects a preamble being transmitted, it keeps receiving it until it decodes the data packet. A more advanced version of preamble sampling is SCP-MAC in which nodes synchronize on a common sleep/wake schedule to reduce the overhead of sending long preambles.

Our energy model takes into account the energy drained by the radio, which includes the energy drained in transmission and reception of packets as well as that drained while listening to the channel periodically checking for incoming traffic.

We propose to evaluate the mean power consumption of the three temperature aware algorithms proposed above and compare them to a reference protocols which we call Default. We also consider the flooding as the traffic model where each node forwards the message it receives exactly once. The summary of the various parameters used in the evaluation is presented in Table 1.

\subsection{Algorithms overall behavior}

In our settings, the initial deployment was optimized so that at temperature $\tau^{+}=80^{\circ} \mathrm{C}$ nodes use that 

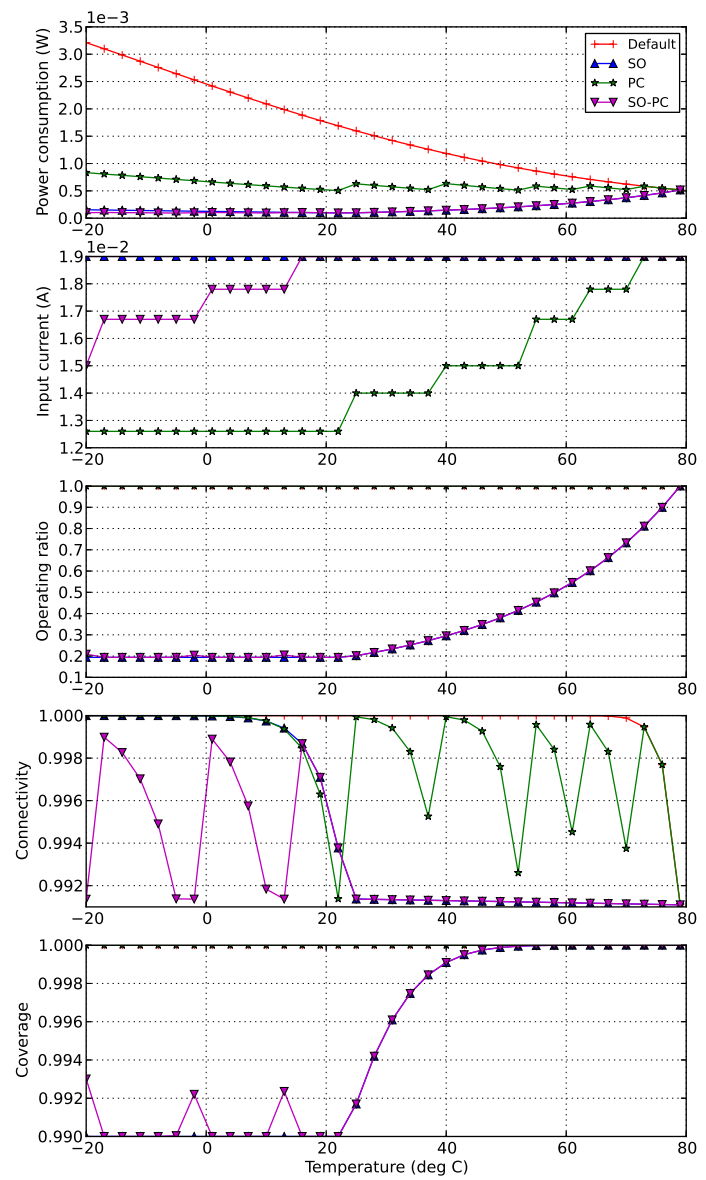

Fig. 5. Numerical Evaluation on BMAC.

maximum ${ }^{4}$ input current $I^{+}=19 \mathrm{~mA}$. Figures 5 and 6 show that both BMAC and SCP-MAC generally exhibit the same overall behavior, which we also expect to be the same for most similar low power MAC protocols, as explained below.

According to our initial deployment, we show that all four algorithms: Default, SO, PC, and SOPC consume the same amount of power in the worst case as all nodes need to be operating normally and transmitting with the maximum power. For the Default algorithm, the mean power consumed increases when temperature decreases. This is due to the increase of transmission range caused by temperature decrease, which make each node spend more energy in receiving more packets as each node

4. We chose to use the maximum input current to minimize the number of deployed nodes. If the number of deployed nodes is not of great importance and the energy consumption model and traffic patterns are known, we can find the optimal input current to be used, and the corresponding number of nodes, so that the correct operation of the network is guaranteed with minimum overall power consumption in the worst case.
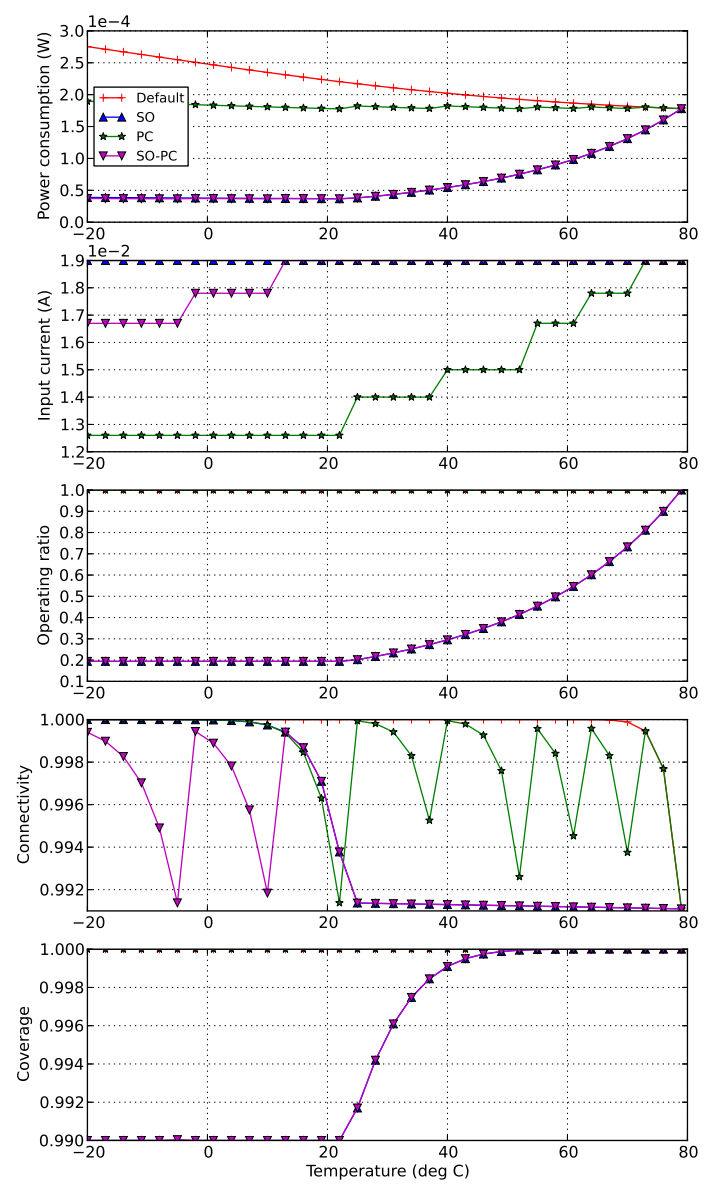

Fig. 6. Numerical Evaluation on SCP.

has a larger number of neighbors when temperature decreases.

In the PC algorithm, the overall power consumption also continues to increase when temperature decreases, as explained in the paragraph above for the Default protocol. However, that increase only continues up to a point when the decrease of temperature is enough to allow the using of a smaller input current that is large enough to continues to preserve the correct operation of the network. When a smaller input current is used, the transmission range decreases compared to the precedent ones before shifting to lower input current, and thus results in the decrease of the overall power consumption. This fluctuation, increase and then decrease in mean power consumption, is observed until no smaller input currents preserving the correct operation of the network can be used. Note that if the input current was given as a continue function, the overall power consumption would decrease slightly when the temperature decreases until the minimum input current that guarantees the 
correct network operation is reached. This is so, because the target communication range is fixed and the PC algorithm will always seek using smaller input current to save more energy. When no smaller input currents can be used and temperature continues to decrease, the transmission range increases and so will the number of neighbors thus resulting in an increase in the mean power consumption.

Contrary to Default and PC algorithms, the overall power consumption always decreases when temperature decreases in the case of the SO algorithm. The decrease of overall power consumption is caused by the decrease of the number of nodes that continue to operate normally to preserve the correct operation of the network. Note that there will be no increase in overall power consumption caused by larger transmission range, because the effective node density remains the same, which the minimum one to preserve the correct operation of the network. The SO algorithm provides significant power savings; however, it is not always possible to constantly put more nodes into hibernate mode when temperature decreases. The subplots about coverage in Figures 5 and 6 show that below $20^{\circ} \mathrm{C}$ it is not possible to put more nodes into hibernate mode anymore because the effective node density will not be high enough to preserve the network coverage (higher than $99 \%$ ).

In the SOPC algorithm, it is generally more energy saving to put more nodes into hibernate mode than to use lower input current. Although this is settingsspecific and depends on many parameters such as MAC protocols, hardware specifications, and traffic load, we expect it to hold for most WSN settings because the power drained in hibernate mode is very low compared to when nodes are other active modes. We also show that amount of gain in energy savings depends on the performance of the MAC protocol used; the more energy-efficient the MAC protocol, the smaller the gain obtained, as shown in figures 5 and 6 where the gain by the use of SO is higher in BMAC than SCP-MAC. This is so, because in very efficient MAC protocols the amount of time nodes spend in sleep mode is already very high so much so there is less room for improvement.

Although the SO part of the SOPC algorithm allows higher energy savings than the PC part, it has limitations, which occur when the correct operation of the network becomes determined by the guarantee of coverage. With the coverage range used in our configuration, this happens when temperature drops below $20^{\circ} \mathrm{C}$. Below that temperature, it is not possible to put more nodes in hibernate mode because the coverage will no longer be maintained. In such a situation, the only possible way to save energy is to use smaller transmission powers which will continue to maintain coverage. The chosen transmission power to be used should continue to maintain connectivity. The using of smaller input current shows the benefit of SOPC over SO, which is noticed when temperature drops below $20^{\circ} \mathrm{C}$. The small fluctuations observed with SOPC are due to the PC part of the SOPC

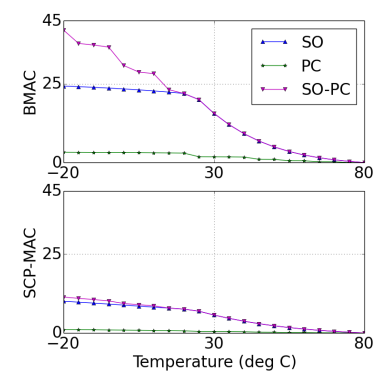

(a)

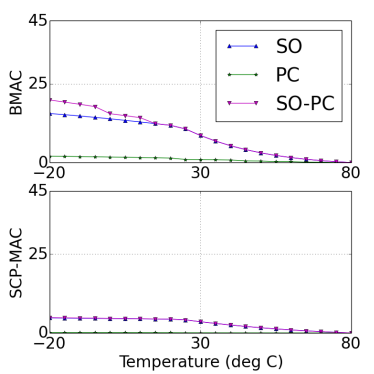

(b)
Fig. 7. describes the energy savings obtained with our algorithms on BMAC and SCP-MAC with two different inter data packet intervals (100 seconds in (a) and 1000 seconds in (b))

algorithm which has been explained above. Note that in some cases, it is better to use lower input current and put less nodes into hibernate mode as shown with BMAC in Figure 5 when temperature drops below $20^{\circ} \mathrm{C}$.

The subplots about the input current that is optimal in the case of SOPC in figures 5 and 6 show that it is more energy saving to use higher input currents in SCPMAC and put more nodes into hibernate mode. This is not the case for BMAC where it is more energy saving to further reduce the input current. The optimal input current depends on many other parameters such as the traffic and power consumption of the various modes (sleep, transmit, receive) of the radio chip.

\subsection{Effect of Traffic Load}

In figures $7(a)$ and $7(b)$, we study the effect of varying the traffic load on the performance of the proposed algorithms. The plots show that higher gains are obtained with less efficient MAC protocols. In fact, BMAC is less efficient in managing energy consumption compared to SCP-MAC which allows nodes to spend more time in sleep mode by avoiding the using of long preambles (see [14]). Therefore there is more room for improvement with BMAC. In addition, the higher the traffic load in the network the more energy efficient are the proposed algorithms, because higher traffic loads involve more energy to be spent in exchanging data where our optimizations operate. However, in low traffic loads most of the power is drained in the idle listening as nodes need to do periodic channel sampling to check for incoming traffic, therefore our optimizations particularly the one involving using lower input current will not have any effect when there is no traffic.

\subsection{Effective duty cycle / settings efficiency}

To evaluate the amounts of energy saving obtained with each algorithm of the three proposed algorithms 
TABLE 2

Mean power consumption for each technique

\begin{tabular}{ll}
\hline \hline $\mathcal{Q}_{\tau}\left(I^{+}, 1\right)$ & Mean power with Default \\
$\mathcal{Q}_{\tau}\left(I^{+}, \tilde{\rho}_{\tau}\left(I^{+}\right)\right)$ & Mean power drained with SO \\
$\mathcal{Q}_{\tau}(\tilde{I}, 1)$ & Mean power drained with PC \\
$\mathcal{Q}_{\tau}\left(\tilde{I}, \tilde{\rho}_{\tau}(\tilde{I})\right)$ & Mean power drained with SOPC \\
\hline \hline
\end{tabular}

compared to the Default algorithm, we calculate the ratio $\mathcal{G}_{\tau}^{+}(I, \rho)$ defined as:

$$
\mathcal{G}_{\tau}^{+}(I, \rho)=\frac{\mathcal{Q}_{\tau}\left(I^{+}, 1\right)-\mathcal{Q}_{\tau}(I, \rho)}{\mathcal{Q}_{\tau}(I, \rho)}
$$

where $\mathcal{Q}_{\tau}(I, \rho)$ is overall power consumption. See Table 2 for the values of $\mathcal{Q}_{\tau}(I, \rho)$ for the four considered algorithms.

The gain achieved by each algorithm depends on the efficiency of the initial setting of the WSN which includes the efficiency of the MAC protocol used, the hardware used, and traffic patterns. Higher energy savings compared to the Default algorithm are achieved when the initial setting is less efficient. To quantify the efficiency of an initial setting, we define the effective duty cycle $\delta$ as the ratio between the mean power $\mathcal{P}_{h}$ drained in hibernate mode to the mean power $\mathcal{Q}_{\tau}\left(I^{+}, 1\right)$ drained by the default algorithm (see Table 2 for the mean power drained by each algorithm). Therefore, we have:

$$
\delta=\frac{\mathcal{P}_{h}}{\mathcal{Q}_{\tau}\left(I^{+}, 1\right)}
$$

Typically the value of $\mathcal{P}_{h}$ is much smaller than $\mathcal{Q}_{\tau}\left(I^{+}, 1\right)$; therefore, the effective duty cycle cannot be larger than 1 . By replacing $\mathcal{Q}_{\tau}\left(I^{+}, 1\right)$ by its value calculated from (47), we obtain:

$$
\delta=\frac{\mathcal{P}_{h}}{\mathcal{P}_{\tau}\left(I^{+}, 1\right)}
$$

In the case of the $\mathrm{SO}$ algorithm, the gain is $\mathcal{G}_{\tau}^{+}\left(I^{+}, \tilde{\rho}_{\tau}\left(I^{+}\right)\right)$. By replacing $\mathcal{Q}_{\tau}\left(I^{+}, 1\right)$ and $\mathcal{Q}_{\tau}(I, \rho)$ with their values from (47), (51) can be rewritten as:

$$
\mathcal{G}_{\tau}^{+}\left(I, \tilde{\rho}_{\tau}\left(I^{+}\right)\right)=\frac{1-\tilde{\rho}_{\tau}\left(I^{+}\right)}{\tilde{\rho}_{\tau}\left(I^{+}\right)+\delta /(1-\delta)}
$$

The expression of the gain above (54) can be rewritten by making abstraction of the temperature and the current and considering the effective duty cycle $\delta$ and the operating ratio $\rho$. Therefore, we have:

$$
\mathcal{G}_{\delta, \rho}=\frac{1-\rho}{\rho+\delta /(1-\delta)}
$$

As the values of $\delta$ and $\rho$ are both belong to the interval $(0,1]$, Equation (55) provides and insight about the theoretical limitations about the gain that be achieved in function of the effective duty cycle. For an optimal configuration, i.e. highly efficient hardware, optimized

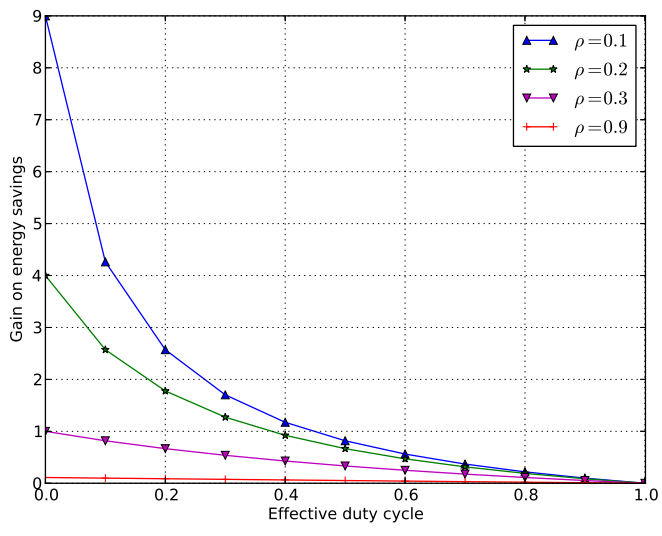

Fig. 8. Limits of the gain on energy savings with the SO algorithm.
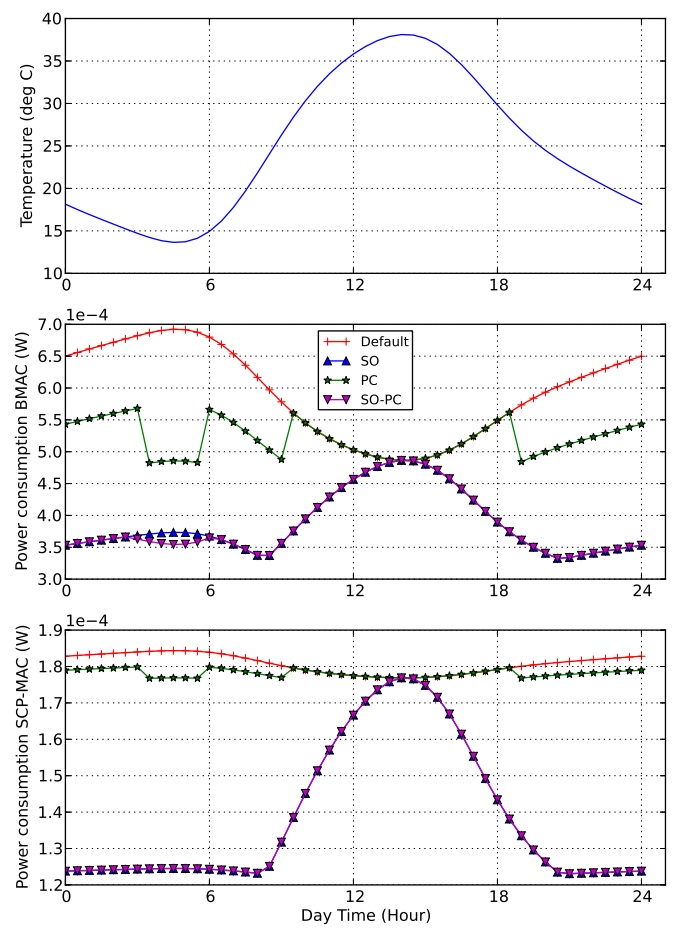

Fig. 9. Mean power consumption with Erbs model.

MAC protocol, and low traffic, the value of $\delta$ will be close to 1 as the mean power drained during the operation of the network is too small and close to that drained in hibernate mode. In this case, the gain obtained is close to zero and the gain by any additional protocols is negligible. However, when $\delta$ is close to zero which indicates a non optimal configuration, the limit of the gain is bounded by $(1-\rho) / \rho$. Buy assuming different values for $\delta$ to reflect different settings, and different values for $\rho$ to reflect different protocol operations for 
different temperatures, we plot Figure 8 which shows the limits of the gain in function of $\delta$ with different values for $\rho$.

\subsection{Using Temperature Variations Model}

We use the model developed by Erbs [19], [20] which shows daily temperature variations. In Erbs models, derived in [21], the average normalized diurnal temperature variation is represented by:

$\tau(y, m, t)=\tau(y, m)+A_{\tau}(y, m) \sum_{n=1}^{4} a_{n} \cos \left(n \gamma(t)-b_{n}\right)$

where $\gamma(t)=2 \pi(t-1) / 24 ; t$ is the time in hour; $\tau(y, m)$ is the daily monthly mean air temperature; and $A_{\tau}(y, m)$ is the monthly mean thermal amplitude expressed in ${ }^{\circ} \mathrm{C}$ and $a_{n}, b_{n}$ are constants which can be found in [21]. For the same month $m$ and the same year $y, \tau(y, m)$ and $A_{\tau}(y, m)$ are constants which we take to be equal to $25^{\circ} \mathrm{C}$. Therefore, we drop the $y$ and $m$ parameters for the sake of simplicity and use $\tau(t)$ to refer to temperature variations in function of time $t$ of the day. In Fig. 9 (top), we plot the daily temperature variation for $\tau(y, m)=A_{\tau}(y, m)=25^{\circ} \mathrm{C}$. The other plots (middle and bottom) present the mean power consumption of the four algorithms (Default, SO, PC, and SOPC) with both BMAC and SCP-MAC protocol and show that gain is significant during lower temperature times which happens at night where temperature drops from above $35^{\circ} \mathrm{C}$ to below $15^{\circ} \mathrm{C}$.

\subsection{Effect of Temperature Sensor Accuracy}

There are many temperature sensors that can equip sensor nodes. These sensors have different accuracies. For example, the STS21 [22] offers an accuracy of less than $\pm 1^{\circ} \mathrm{C}$ in the range of $\left[-40^{\circ} \mathrm{C}, 100^{\circ} \mathrm{C}\right]$. Another example is the SHT11 [23] which has an accuracy of $\pm 2.5^{\circ} \mathrm{C}$ in the range of $\left[-40^{\circ} \mathrm{C}, 100^{\circ} \mathrm{C}\right]$. The highest accuracy is around the typical temperature of $25^{\circ} \mathrm{C}$ and the more we move out of the typical temperature, the accuracy reduces. From the data provided in [23] for the SHT11 temperature sensor, we can model the variation in temperature as follows:

$$
\Delta \tau=0.03 \times|\tau-25|+0.35
$$

where $\Delta \tau$ is the variation in temperature and $\tau$ is the real temperature. The measured temperature $\hat{\tau}$ will be in the interval $[\tau-\Delta \tau, \tau+\Delta \tau]$. By considering the model described in (57), the real temperature $\tau$ will belong to the interval described below.

$$
(\hat{\tau}+0.4) / 1.03<\tau<(\hat{\tau}-0.4) / 0.97
$$

From (58), we can obtain the worst and the best case values for the real temperature $\tau$ given the measured temperature $\hat{\tau}$. If the real temperature is smaller than the measured temperature, our algorithms will continue to preserve the correct operation of the network but the

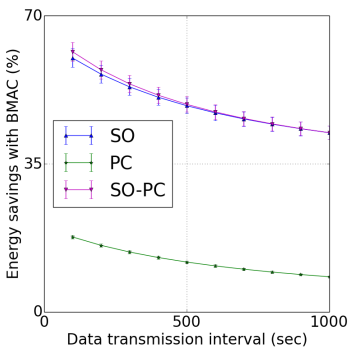

(a)

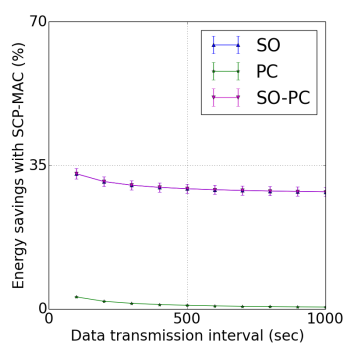

(b)
Fig. 10. (a) Mean power consumption with Erbs model (BMAC). (b) Mean power consumption with Erbs model (SCP-MAC).

amount of the obtained gain will not be as good as if the exact value of the real temperature was known. However, if the real temperature is higher than the measured temperature, our algorithms will not operate properly. Therefore, our algorithms need to consider this inaccuracy in the worst case and use the upper value for temperature $(\hat{\tau}-0.4) / 0.97$ in execution of the algorithms SO, PC, and SOPC. Fig. 10(a) and Fig. 10(b) show the effect of temperature reading inaccuracies on the amount of the gain obtained. The error bars on these figures reflect the gain that can be achieved in the worst, average, and best cases. We show that even by operating our algorithms in the worst case the gain is still substantial.

\subsection{Overall power consumption}

Fig. 10(a) and Fig. 10(b) show the percentage of energy saving that can be made by using one of the three algorithms (SO, PC, and SOPC) when the temperature varies according to the Erbs model. Fig 10(a) show that more energy savings can be made with BMAC compared to those that can be made with SCP-MAC shown in Fig 10(b). This confirms that our algorithms achieve fewer savings under efficient configuration. However, the savings obtained are still significant nearly independently of traffic load. With SCP-MAC, our most efficient algorithms (SO and SOPC) achieve around 30\% of savings with varying traffic load from one packet every 100 s to one every 1000 s.

\section{Analogy to topology Control algo- RITHMS}

By putting nodes in hibernate mode or letting them use different transmission powers, our algorithms (SO, PC, and SOPC) affect the topology of the entire network as some links may appear and others disappear. As such our algorithms may share some aspects with other topology control solutions. However, there are also other aspects that make our solutions distinguishable particularly taking into account the effect of temperature on link quality. 
In addition, existing topology control solutions such as LMST, RNG, etc. [24] rely on information exchange among nodes to build a local or global view of the network topology. This is typically realized through the exchange of hello messages to maintain a 1-hop, 2-hop, ..., or a k-hop topology. The overhead of such message exchange may increase substantially with the size of the topology view that needs to be maintained, because more links need to be assessed and some links may require a number of transmissions before a final decision can be made about whether to include the link or not.

Furthermore, a significant part of research carried out in the area of topology control is based on deterministic radio model and the network is modeled by a graph where a link between two nodes exists if the distance separating those node is smaller than the assumed communication range. With this assumption, the solutions proposed targeted achieving 100\% connectivity. In real environments, the link between nodes are probabilistic and every message transmission is related to a probability that mainly depends on distance in stable radio environments. Probabilistic Topology Control (PTC) has been proposed in [25]. The PTC relies on the quality of connectivity that is measured by network reachability, which is defined as the minimal end-to-end delivery ratio between any two pair of nodes using a network wide broadcast. The paper showed that the energy cost to achieve a certain network reachability is significantly higher with deterministic topology control than with the probabilistic one. The paper is, however, focused on finding the minimal transmission power and thus is less efficient than power mode protocols as shown by our analysis in this paper. It assumes that the quality of links is available but it does assess the cost of realizing such assumption which could be potentially high. The authors also assume that link qualities are fixed and therefore do not take into account the dynamic case where link qualities can be affected by changing parameters such as temperature.

Finally, unlike most of traditional topology control solutions, our algorithms integrate both connectivity and coverage which are the two requirements for the correct operation of the network. Previous solutions either use power control which maintains the coverage but does not provide optimal energy savings, or focus on connectivity and put node into sleep mode to achieve high energy saving without jointly considering the coverage problem that occurs when too many nodes put into sleep mode.

\section{Conclusions}

We showed that temperature variations significantly affect the quality of wireless links in an almost deterministic way. A reliable deployment needs to take the worst case into account, particularly when temperature variations are significant from days to nights, and from a season to another. When networks are deployed according to the worst case, algorithms should be designed to optimize the operation of network when conditions improve. We designed our algorithms along this line with the focus on making them simple and effective. We proposed and evaluated three algorithms SO (StopOperate), PC (Power-Control), and SOPC (Stop-Operate and Power-Control). All of the algorithms target improving the energy efficiency while maintaining their correct operation by ensuring both connectivity and coverage are preserved. Our algorithms have been optimized for stable radio environments where significant link variations are governed by a deterministic pattern such as the temperature effect on link quality. They have been designed with an integrated connectivity/coverage support and taking into account the probabilistic nature of the wireless channel as opposed to deterministic topology control solutions. In addition, they do not rely on any information or message exchange between nodes which increases their robustness and efficiency compared to other neighborhood based topology control algorithms.

Our results show that in typical sensor network configurations the $\mathrm{SO}$ algorithm achieves higher energy savings than the PC one. However, the SO algorithm cannot be used in all situations as it affects both connectivity and coverage. In some situations where the coverage will be affected by putting further nodes in stop mode, the PC algorithm should be used along with the $\mathrm{SO}$ one to achieve further energy savings, as proposed in the SOPC algorithm.

The current work is limited to the case where nodes are deployed randomly with a constant and homogeneous density over the deployment area. These assumptions require relaxation and further investigation should be carried out to study the case of regular deployment and the case where the deployment is not homogeneous.

\section{ACKNOWLEDGMENT}

This work is funded in part by the Algerian Ministry of Higher Education and Scientific Research under contract B*01420130132, Imperial College London, the French Government, through the program Investments for the future managed by the National Agency for Research (Reference ANR-11-IDEX-0004-02), and under the framework of the Labex MS2T.

Special thanks go to the reviewers for their comments and useful suggestions that have significantly improved the readability of this survey paper.

\section{REFERENCES}

[1] Chipcon Corporation. CC2400 2.4 GHz Low-Power RF Transceiver, Data Sheet, 2008.

[2] Texas Instruments. CC2530 A True System-on-Chip Solution for 2.4-GHz IEEE 802.15.4 and ZigBee Applications, Data Sheet, 2009.

[3] J. Otero, P. Yalamanchili, and H.-W. Braun. High performance wireless networking and weather. Technical report, University of California, San Diego, 2001.

[4] C. A. Boano, J. Brown, Z. He, U. Roedig, and T. Voigt. Lowpower radio communication in industrial outdoor deployments: The impact of weather conditions and atex-compliance. In SENSAPPEAL, pages 159-176, 2009. 
[5] C. A. Boano, J. Brown, N. Tsiftes, U. Roedig, and T. Voigt. The impact of temperature on outdoor industrial wsn applications. IEEE Trans. Industrial Informatics, 6(3):451-459, 2010.

[6] Jungwook Lee and Kwangsue Chung. An efficient transmission power control scheme for temperature variation in wireless sensor networks. Sensors, 11:3078 - 3093, 2011.

[7] Jung Zhang, Guifang Qiao, Zhen Li, and Weiguo Wang. Future Wireless Networks and Information Systems, volume LNEE 144, chapter The Impact of Ambient Temperature Variations on Signal Intensity of Outdoor WSN System, pages 79-86. Springer, 2012.

[8] Kenneth Bannister, Gianni Giorgetti, and Sandeep Gupta. Wireless sensor networking for hot applications: Effects of temperature on signal strength, data collection and localization. In Proceedings of the 5nd IEEE workshop on Embedded Networked Sensors, Charlotteville, VA, USA, 2008.

[9] Nouha Baccour, Anis Koubaa, Habib Youssef Marco Zuniga, Carlo Alberto Boano, and Mario Alves. Radio link quality estimation in wireless sensor networks: a survey. ACM Trans. Sen. Netw., 8, November 2012.

[10] Theodore Rappaport. Wireless Communications: Principles and Practice. Prentice Hall PTR, Upper Saddle River, NJ, USA, 2nd edition, 2001

[11] Peter Hall. Introduction to the Theory of Coverage Processes. J. Wiley and Sons, NY, USA, 1988.

[12] D. Miorandi. The impact of channel randomness on coverage and connectivity of ad hoc and sensor networks. Trans. Wireless. Comm., 7(3):1062-1072, March 2008.

[13] R. M. Corless, G. H. Gonnet, D. E. G. Hare, D. J. Jeffrey, and D. E. Knuth. On the lambert $\mathrm{w}$ function. In ADVANCES IN COMPUTATIONAL MATHEMATICS, pages 329-359, 1996.

[14] Wei Ye, Fabio Silva, and John Heidemann. Ultra-low duty cycle mac with scheduled channel polling. In Proceedings of ACM SenSys, pages 321-334, 2006.

[15] IEEE 802.15.4. Wireless Medium Access Control (MAC) and Physical Layer (PHY) Specifications for Low-Rate Wireless Personal Area Networks (LR-WPANs), 2003.

[16] D. Stoyan, W. S. Kendall, and J. Mecke. Stochastic Geometry and its Applications. J. Wiley and Sons, NY, USA, 1987.

[17] Joseph Polastre, Jason Hill, and David Culler. Versatile low power media access for wireless sensor networks. In Proceedings of the 2nd international conference on Embedded networked sensor systems, SenSys '04, pages 95-107, New York, NY, USA, 2004. ACM.

[18] A. Bachir, M. Dohler, T. Watteyne, and K. K. Leung. Mac essentials for wireless sensor networks. Commun. Surveys Tuts., 12(2):222248, April 2010.

[19] D. G. Erbs, S. A. Klein, and W. A. Beckman. Estimation of degreeday and ambient temperature bin data from monthlyaverage temperatures. ASHRAE J., 1983.

[20] D. G. Erbs. Models and applications for weather statistics related to building heating and cooling loads. $\mathrm{PhD}$ thesis, Mechanical Engineering Dept., University of Wisconsin Madison, 1984.

[21] Julia Bilbao, Argimiro H. De Miguel, and Harry D. Kambezidis. Air temperature model evaluation in the north mediterranean belt area. J. of applied meteorology, 41:872-884, 2002.

[22] Sensirion. Datasheet STS21 Temperature Sensor IC, Version 2, December 2011.

[23] Sensirion. Datasheet SHT1x (SHT10, SHT11, SHT15) Humidity and Temperature Sensor IC, December 2011.

[24] A. A. Aziz, Y. A. Sekercioglu, P. Fitzpatrick, and M. Ivanovich. A survey on distributed topology control techniques for extending the lifetime of battery powered wireless sensor networks. Commun. Surveys Tuts., 15(1):121-144, 2013.

[25] Yunhuai Liu and Lionel M. Ni. A generalized probabilistic topology control for wireless sensor networks. IEEE Jounal on Selected Areas in Communications, 30(9):1780-1788, 2012.

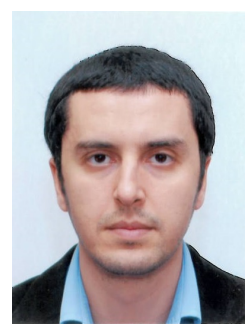

Abdelmalik Bachir graduated from National Institute of Informatics, Algiers, Algeria, in 2001, received the DEA diploma in Informatics from the University of Marseille, France, in 2002 and the Ph.D. degree from Grenoble Institute of Technology, France, in 2007. He took research positions at Avignon University, France Telecom R\&D, and Grenoble Institute of Technology, France. He has also been a research associate at Imperial College London, UK, for a number of years. Currently he is an associate professor at Biskra University, Algeria, and a visiting researcher at Imperial College London, UK. His research interests include: MAC and Routing protocols for wireless networks, user mobility profiling, and inter vehicle communication.

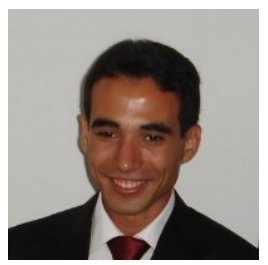

Walid Bechkit is an assistant professor with the Telecommunications department at INSA de Lyon. He is also a member of the INRIA UrbaNet team of the CITI laboratory. He obtained his Ph.D. in System and Information Technology from the Compiegne University of Technology (U.T.C.) in 2012 and his engineering degree in Computer Science from the National School of Computer Science of Algiers (ESI) in 2009. His main research interests include energy saving, reliability and security issues in wireless sensor

networks.

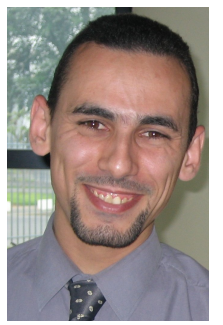

Yacine Challal is associate professor at Compiègne University of Technology (France). $\mathrm{He}$ is member of the "Networking and optimization" research team at the CNRS Heudiasyc Lab. $\mathrm{He}$ got his PhD and Master degrees respectively in 2005, and 2002 from Compigne University of Technology. His research interests include security in group communication, security in wireless mobile networks, wireless sensor networks, IoT, Cloud computing and fault tolerance in distributed systems.

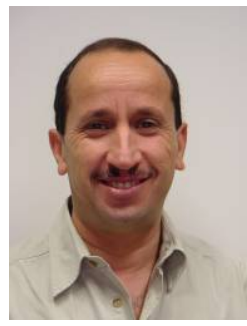

Abdelmadjid Bouabdallah received the Engineering degree from USTHB-Algeria, Master degree in 1988 and Ph.D. from university of Paris-sud Orsay (France) in 1991. From 1992 to 1996, he was Assistant Professor at university of Evry-Val-d?Essonne (France) and since 1996 he is Professor at University of Technology of Compiegne (UTC) where he is leading the Networking \& Security research group and the Interaction \& Cooperation research of the Excellence Research Center LABEX MS2T. His research interest includes Internet QoS, security, unicast/multicast communication, wireless sensor networks, and fault tolerance in wired/wireless networks. He conducted several large scale research projects funded by well known companies (Motorola Labs., Orange Labs., CEA, etc.) as well as academic organizations (ANR-RNRT, CNRS, ANR-Carnot). 